Article

\title{
Antimicrobial, Antioxidant, and Cytotoxic Activities of Ocimum forskolei and Teucrium yemense (Lamiaceae) Essential Oils
}

\author{
Nasser A. Awadh Ali ${ }^{1,2, *}$, Bhuwan K. Chhetri ${ }^{3}$, Noura S. Dosoky ${ }^{3}$, Khola Shari ${ }^{2}$, \\ Ahmed J. A. Al-Fahad ${ }^{4}$, Ludger Wessjohann ${ }^{5}$ and William N. Setzer ${ }^{3, *}$ \\ 1 Department of Pharmacognosy, Faculty of Clinical Pharmacy, Albaha University, P.O. Box 1988, \\ Albaha, Saudi Arabia \\ 2 Department of Pharmacognosy and Phytochemistry, Faculty of Science, Sana'a University, P.O. Box 13150, \\ Sana'a, Yemen; sharee_8855@yahoo.com \\ 3 Department of Chemistry, University of Alabama in Huntsville, Huntsville, AL 35899, USA.; \\ bhuwanchhetri89@gmail.com (B.K.C.); nouradosoky@yahoo.com (N.S.D.) \\ 4 Department of Chemistry, Faculty of Science, Albaha University, P.O. Box 2345, Albaha, Saudi Arabia; \\ aalfahad@bu.edu.sa \\ 5 Leibniz Institute of Plant Biochemistry, Department of Bio-Organic Chemistry, Weinberg 3, \\ 06120 Halle, Germany; wessjohann@ipb-halle.de \\ * Correspondence: alinasser9678@yahoo.com (N.A.A.A.); wsetzer@chemistry.uah.edu (W.N.S.); \\ Tel.: +966-05-0308-5786 (N.A.A.A.); +1-256-824-6519 (W.N.S.)
}

Academic Editor: Eleni Skaltsa

Received: 25 February 2017; Accepted: 29 March 2017; Published: 1 April 2017

\begin{abstract}
Background: Ocimum forskolei and Teucrium yemense (Lamiaceae) are used in traditional medicine in Yemen. Methods: The chemical composition, antimicrobial, antioxidant and cytotoxic activities of the essential oils isolated from the leaves of Ocimum forskolei Benth. (EOOF) and two different populations of Teucrium yemense Deflers., one collected from Dhamar province (EOTY-d), and another collected from Taiz (EOTY-t) were investigated. The antimicrobial activities of the oils were evaluated against several microorganisms with the disc diffusion test or the broth microdilution test. The essential oils were screened for in-vitro cytotoxic activity against human tumor cells. EOOF and EOTY-d were screened for free-radical-inhibitory activity using the DPPH radical scavenging assay. Results: Sixty-four compounds were identified in (EOOF) representing $100 \%$ of the oil content with endo-fenchol (31.1\%), fenchone (12.2\%), $\tau$-cadinol (12.2\%), and methyl (E)-cinnamate $(5.1 \%)$ as the major compounds. In EOTY-d, 67 compounds were identified, which made up $91 \%$ of the total oil. The most abundant constituents were $(E)$-caryophyllene $(11.2 \%), \alpha$-humulene (4.0.\%), $\gamma$-selinene (5.5\%), 7-epi- $\alpha$-selinene $(20.1 \%)$, and caryophyllene oxide $(20.1 \%)$, while the major compounds in EOTY-t were $\alpha$-pinene $(6.6 \%),(E)$-caryophyllene $(19.1 \%) \alpha$-humulene $(6.4 \%)$, $\delta$-cadinene $(6.5 \%)$, caryophyllene oxide $(4.3 \%), \alpha$-cadinol (9.5\%), and shyobunol (4.6\%). The most sensitive microorganisms for EOOF were B. subtilis, S. aureus, and C. albicans with inhibition zones of 34,16 , and $24 \mathrm{~mm}$ and MIC values of, $4.3 \mathrm{mg} / \mathrm{mL}, 4.3 \mathrm{mg} / \mathrm{mL}$, and $8.6 \mathrm{mg} / \mathrm{mL}$, respectively. EOTY-t showed antimicrobial activity against $S$. aureus, B. cereus, A. niger, and B. cinerea with MIC values of $0.156,0.156,0.313$ and $0.313 \mathrm{mg} / \mathrm{mL}$, respectively. Neither essential oil showed remarkable radical inhibition $\left(\mathrm{IC}_{50}=31.55\right.$ and $31.41 \mu \mathrm{L} / \mathrm{mL}$ ). EOTY-d was active against HT-29 human colorectal adenocarcinoma cell lines with $\mathrm{IC}_{50}=43.7 \mu \mathrm{g} / \mathrm{mL}$. Consistent with this, EOTY-t was active against both MCF-7 and MDA-MB-231 human breast adenocarcinoma cells. Conclusions: The antimicrobial activity of Ocimum forskolei essential oil against B. subtilis and C. albicans is consistent with its traditional use in Yemeni traditional medicine to treat skin infections. Both O. forskolei and T. yemense show wide variations in their respective essential oil compositions; there remains a need to investigate both species botanically, genetically, and phytochemically more comprehensively.
\end{abstract}


Keywords: antibacterial; antifungal; free radical scavenging; antineoplastic

\section{Introduction}

Ocimum forskolei Benth. (Lamiaceae) is one of about 65 tropical and subtropical species of Ocimum, seven of which are found in Yemen, and these include O. basilicum L., O. tenuiflorum L., O. suave Willd., O. spicatum Deflers, O. gratissimum L., and O. forskolei [1]. Several species of Ocimum are aromatic and used as flavoring agents, insect repellents, and traditional medicines [2]. O. forskolei ranges in East Africa from Egypt, south to Kenya [3], and the southern Arabian Peninsula, including Yemen [4], Oman [5], and the United Arab Emirates (UAE) [6]. O. forskolei is used traditionally in Yemen as a cosmetic, to relieve fever, and to treat skin infections [7]. In UAE, crushed leaves of O. forskolei are used to treat headaches, colds, and ear aches, while the juice is used as eye drops or for insect bites [6]. The plant is used in Eritrea as a mosquito repellent and has demonstrated repellent activity [8]. The dichloromethane extract of $O$. forskolei has shown weak antibacterial activity against Gram-positive bacteria, while the methanol extract showed minimal radical-scavenging activity [7]. Previous investigations of $O$. forskolei have shown the essential oil to have weak antioxidant [9] and nematicidal activity [10], and better activities against bacteria and dermatophytes [11].

Teucrium is a large genus belonging to the Lamiaceae which contains about 250 species that are widespread over the world, but are most common in Mediterranean climates [2]. Teucrium species endemic to Yemen are T. yemense Deflers, T. socotranum (Balf. f.) Vierh., and T. balfourii (Balf. f.) Vierh. Plants from this genus are perennial in the form of herbs, shrubs, and sub-shrubs. Teucrium species are widely used in Yemeni folk medicine as antispasmodics and insect repellants [12]. Teucrium species are rich in essential oil and are valued as ornamental plants. Teucrium yemense is mostly found in Djibouti, Ethiopia, Saudi Arabia, Sudan, and Yemen. It is a perennial aromatic plant possessing sessile oblanceolate leaves and dense terminal heads of white flowers [12]. Essential oil studies of various species of Teucrium have shown $\alpha$-humulene, $\delta$-cadinene, $(E)$-caryophyllene, $\alpha$-pinene, $\beta$-pinene, linalool, 3-octanol, $\alpha$-cadinol, caryophyllene oxide, 8-cedren-13-ol, and (E)- $\beta$-farnesene to be the major components of the essential oils. A wide range of biological activity has been reported for Teucrium species, some of which are attributed to the high content of essential oil in them. They possess antifungal, antibacterial, larvicidal, antispasmodic, antioxidant, anti-inflammatory, antiulcer, hypoglycemic, antiacetylcholinesterase, and hepatoprotective activities [12].

\section{Materials and Methods}

\subsection{Plant Materials}

The leaves of $O$. forskolei and $T$. yemense were collected from several mature plants during the flowering stage in May 2010 from Dhamar province, Yemen. The plants were identified by Hassan M. Ibrahim of the Botany Department, Faculty of Sciences, Sana'a University. Voucher specimens (YMP Lam-33 and YMP Lam-36) have been deposited at the Pharmacognosy Department, Sana'a University, Yemen. Another sample of T. yemense leaves was collected in February 2012 from Taiz town, Yemen. For each sample, the dried leaves ( $100 \mathrm{~g})$ were hydrodistilled for $3 \mathrm{~h}$ in a Clevenger type apparatus according to the European Pharmacopoeia method [13]. The obtained oils were subsequently dried over anhydrous $\mathrm{Na}_{2} \mathrm{SO}_{4}$ and kept at $4{ }^{\circ} \mathrm{C}$ until analysis.

\subsection{Gas Chromatography-Mass Spectrometry (GC-MS)}

O. forskolei and T. yemense essential oils were analyzed by GC-MS using an Agilent 6890 GC with an Agilent 5973 mass selective detector (MSD, operated in the EI mode (electron energy $=70 \mathrm{eV}$ ), scan range $=40-400 \mathrm{amu}$, and scan rate $=3.99 \mathrm{scans} / \mathrm{s}$ ), and an Agilent ChemStation data system. The GC column was an HP- $5 \mathrm{~ms}$ fused silica capillary with a ( $5 \%$ phenyl)-polymethylsiloxane stationary 
phase, film thickness of $0.25 \mu \mathrm{m}$, a length of $30 \mathrm{~m}$, and an internal diameter of $0.25 \mathrm{~mm}$. The carrier gas was helium with a column head pressure of $48.7 \mathrm{kPa}$ and a flow rate of $1.0 \mathrm{~mL} / \mathrm{min}$. Inlet temperature was $200{ }^{\circ} \mathrm{C}$ and interface temperature was $280^{\circ} \mathrm{C}$. The GC oven temperature program was used as follows: $40{ }^{\circ} \mathrm{C}$ initial temperature, held for $10 \mathrm{~min}$; increased at $3{ }^{\circ} \mathrm{C} / \mathrm{min}$ to $200{ }^{\circ} \mathrm{C}$; increased $2^{\circ} / \mathrm{min}$ to $220^{\circ} \mathrm{C}$. A $1 \%(\mathrm{w} / v)$ solution of the sample in $\mathrm{CH}_{2} \mathrm{Cl}_{2}$ was prepared and $1 \mu \mathrm{L}$ was injected using a splitless injection technique. Identification of the oil components was based on their retention indices as determined by reference to a homologous series of $n$-alkanes $\left(C_{8}-C_{40}\right)$, and by comparison of their mass spectral fragmentation patterns with those reported in the literature [14], and stored on the MS library (NIST database (G1036A, revision D.01.00)/ChemStation data system (G1701CA, version C.00.01.080). The percentages of each component are reported as raw percentages based on total ion current without standardization.

\subsection{Radical Scavenging Assay}

For the preliminary test, analytical TLC (thin-layer chromatography) on silica gel plates was developed under appropriate conditions after application of $5 \mu \mathrm{L}$ of oil solution, dried and sprayed with DPPH solution $(0.2 \%, \mathrm{MeOH})$. Five minutes later, active compounds appeared as yellow spots against a purple background. Estimation of a radical scavenging effect was carried out by using a DPPH (2,2-diphenyl-1-picrylhydrazyl) free radical scavenger assay in 96-well microtiter plates (MTPs) according to a modified method [15]. A solution of DPPH (Sigma-Aldrich, Munich, Germany) was prepared by dissolving $5 \mathrm{mg}$ DPPH in $2 \mathrm{~mL}$ of methanol, and the solution was kept in the dark at $4{ }^{\circ} \mathrm{C}$ until use. Stock solutions of the samples were prepared at $2 \mathrm{mg} / \mathrm{mL}$ and diluted to different concentrations. Methanolic DPPH solution $(5 \mu \mathrm{L})$ was added to each well. The plate was shaken for $2 \mathrm{~min}$ to ensure thorough mixing before being wrapped in aluminum foil and stored in the dark. A methanolic solution of DPPH served as control. After $30 \mathrm{~min}$, the optical density (OD) of the solution was measured at a wavelength of $517 \mathrm{~nm}$ using a microtiter plate ELISA reader (Thermo Scientific, Helsinki, Finland) and the percentage decolorization was calculated as an indication of the antioxidant activity of a sample. Each experiment was carried out in triplicate and $\mathrm{IC}_{50}$ (median inhibitory concentration) values were calculated using Origin software (version 7, OriginLab Corp., Northampton, MA, USA). Ascorbic acid (Sigma-Aldrich, Munich, Germany) was used as a positive control.

\subsection{Antimicrobial Assays}

The antimicrobial activities of O. forskolei (EOOF) and T. yemense (EOTY-d) essential oils were evaluated by the agar disc-diffusion method, as previously described [16]. The microorganisms used were Escherichia coli ATCC 10536, Pseudomonas aeruginosa ATCC 25619, Staphylococcus aureus ATCC 29737, Bacillus subtilis ATCC 6633, methicillin-resistant Staphylococcus aureus (MRSA), and Candida albicans ATCC 2091. Müller-Hinton Agar (MHA) (Merck, Darmstadt, Germany) was used for bacterial culture at $37^{\circ} \mathrm{C}$. Sabouraud dextrose agar (Merck, Darmstadt, Germany) was used to cultivate C. albicans.

T. yemense (EOTY-t) essential oil was screened for antibacterial activity against the Gram-positive bacteria Bacillus cereus (ATCC No. 14579) and Staphylococcus aureus (ATCC No. 29213) and the Gram-negative bacteria Pseudomonas aeruginosa (ATCC No. 27853) and Escherichia coli (ATCC No. 10798). The minimum inhibitory concentrations (MICs) were determined using the microbroth dilution technique [17]. Dilutions of the essential oils were prepared in cation-adjusted Mueller Hinton broth (CAMHB) beginning with $50 \mu \mathrm{L}$ of $1 \% w / w$ solutions of oils in DMSO plus $50 \mu \mathrm{L}$ of CAMHB. The oil solutions were serially diluted (1:1) in CAMHB in 96-well plates. Organisms at a concentration of approximately $1.5 \times 10^{8}$ colony-forming units (CFU) $/ \mathrm{mL}$ were added to each well. Plates were incubated at $37^{\circ} \mathrm{C}$ for $24 \mathrm{~h}$, and the final minimum inhibitory concentration (MIC) was determined as the lowest concentration without turbidity. Gentamicin was used as a positive antibiotic control, while DMSO was used as a negative control [17]. 
The microbroth dilution technique was also applied to determine the antifungal activity of EOTY-t essential oil against Aspergillus niger (ATCC No. 16888), Botrytis cinerea (ATCC No. 126943), and Candida albicans (ATCC No. 90028). For Aspergillus niger and Candida albicans, dilutions of the essential oils were prepared in YM broth beginning with $50 \mu \mathrm{L}$ of $1 \% w / w$ solutions of oils in DMSO plus $50 \mu \mathrm{L}$ of YM broth. The oil solutions were serially diluted (1:1) in YM in 96-well plates. YM broth inoculated with A. niger hyphal and Candida albicans culture diluted to a McFarland turbidity of 1.0 were added to each well. Plates were incubated at $37^{\circ} \mathrm{C}$ for $24 \mathrm{~h}$; amphotericin B was the positive control, while DMSO was used as the negative control. The same process was used for $B$. cinerea as well, except that the inoculum suspension was prepared in potato dextrose broth (PDB) and the inoculum size was adjusted with respective broth medium between $1.0 \times 10^{6}$ and $5.0 \times 10^{6}$ spores $/ \mathrm{mL}$ by maintaining the optical density (OD) between 0.1 and 0.2 at $625 \mathrm{~nm}$. Dilutions were carried out in the fungal growth medium (malt extract broth). Combination antifungal cyprodinil-fludioxonil was used as the positive control and DMSO was used as the negative control. The 96-well plates were incubated at $25 \pm 2{ }^{\circ} \mathrm{C}$ for $48 \mathrm{~h}$.

\subsection{Cytotoxicity Assays}

EOOF and EOTY-d were screened for cytotoxicity on HT-29 (human colorectal adenocarcinoma) cells using the XTT assay [18]. HT29 cells were grown in a $5 \% \mathrm{CO}_{2}$ environment at $37{ }^{\circ} \mathrm{C}$ in RPMI 1640 medium without L-glutamine, supplemented with $10 \%$ fetal bovine serum, $1 \%(200 \mathrm{mM})$ L-alanyl-L-glutamine, and 1.6\% HEPES (1 M). Cells were plated into 96-well cell culture plates at $1.5 \times 10^{3}$ cells (HT29) per well. The volume in each well was $100 \mu \mathrm{L}$. After $24 \mathrm{~h}$, supernatant fluid was removed by suction and replaced with $100 \mu \mathrm{L}$ growth medium containing $0.1 \mu \mathrm{L}$ of a DMSO solution of the essential oil, giving final concentrations of 100, 50, 25, and $12.5 \mu \mathrm{g} / \mathrm{mL}$ for each well. Solutions were added to wells in three replicates. Medium controls without cells and DMSO controls $(0.5 \mu \mathrm{L} \mathrm{DMSO} / \mathrm{mL})$ were used. Digitonin $(125 \mu \mathrm{M})$ was used as a positive control [19]. After the addition of oils, plates were incubated for $72 \mathrm{~h}$ at $37^{\circ} \mathrm{C}$ in $5 \% \mathrm{CO}_{2}$; medium was then removed by suction, and $100 \mu \mathrm{L}$ of fresh medium was added to each well. In order to establish percent growth inhibition rates, the XTT assay for cell viability was carried out according to Cell Proliferation Kit II protocol, (Roche Diagnostics, Mannheim, Germany). After colorimetric readings were recorded (Molecular Devices SpectraMAX M5 microplate reader, $490 \mathrm{~nm}$ ), average absorbance, growth inhibition, and standard deviations were determined.

EOTY-t was screened for cytotoxic activity against MCF-7 (human breast adenocarcinoma, estrogen receptor positive) and MDA-MB-231 (human breast adenocarcinoma, estrogen receptor negative) cells using MTT-based cytotoxicity assay [20]. Human MCF-7 (ATCC No. HTB-22) and MDA-MB-231 (ATCC No. HTB-26) breast adenocarcinoma cells were grown in a 3\% $\mathrm{CO}_{2}$ environment at $37^{\circ} \mathrm{C}$ in RPMI-1640 medium, supplemented with $10 \%$ fetal bovine serum, 100,000 units penicillin, and $10.0 \mathrm{mg}$ streptomycin per liter of medium, $15 \mathrm{mM}$ of HEPES, and buffered with $26.7 \mathrm{mM} \mathrm{NaHCO}$, pH 7.35. Cells were plated into 96-well cell culture plates at $2.5 \times 10^{4}$ cells per well. The volume in each well was $100 \mu \mathrm{L}$. After $48 \mathrm{~h}$, supernatant fluid was removed by suction and replaced with $100 \mu \mathrm{L}$ growth medium containing $1.0 \mu \mathrm{L}$ of DMSO solution of the essential oil ( $1 \% w / w$ in DMSO), giving a final concentration of $100 \mu \mathrm{g} / \mathrm{mL}$ for each well. Solutions were added to wells in four replicates. Medium controls and DMSO controls $(10 \mu \mathrm{L} \mathrm{DMSO} / \mathrm{mL})$ were used. Tingenone was used as a positive control [21]. After the addition of compounds, plates were incubated for $48 \mathrm{~h}$ at $37^{\circ} \mathrm{C}$ in $5 \% \mathrm{CO}_{2}$; medium was then removed by suction, and $100 \mu \mathrm{L}$ of fresh medium was added to each well. In order to establish percent kill rates, the MTT assay for cell viability was carried out [22]. After colorimetric readings were recorded (570 nm, using a SpectraMAX Plus microplate reader, Molecular Devices, Sunnyvale, CA, USA), percent kill was calculated. 


\section{Results and Discussion}

\subsection{Essential Oil Compositions}

\subsubsection{Ocimum forskolei}

The chemical composition of the leaf essential oil of O. forskolei (EOOF) is listed in Table 1. A total of 64 compounds were identified, accounting for $100 \%$ of the essential oil composition. The major components were endo-fenchol (31.1\%), fenchone $(12.2 \%)$, $\tau$-cadinol $(12.2 \%)$, and methyl $(E)$-cinnamate $(5.1 \%)$. The essential oil composition of $O$. forskolei from Yemen is remarkably different from that reported by Fatope and co-workers from Oman [11]. These workers found the leaf oil to be composed largely of estragole $(42 \%-78 \%)$ and linalool $(10 \%-16 \%)$, but apparently no fenchone, fenchol, methyl cinnamate, or $\tau$-cadinol. Al-Hajj and co-workers collected the essential oil of O. forskolei from Sana'a, Yemen, but their essential oil analysis is incorrect and cannot be compared [9]. Headspace analysis of $O$. forskolei from Eritrea showed (E)- $\beta$-ocimene, 4-hexen-1-yl acetate, 3-hexenol, 1-octen-3-ol, $\alpha$-copaene, linalool (major), (E)-caryophyllene (major), $\alpha$-humulene, methyl salicylate, and methyl cinnamate [8]. The large discrepancies in essential oil compositions of $O$. forskolei may reflect taxonomic confusion or hybridization $[3,23]$.

Table 1. Leaf essential oil compositions of Ocimum forskolei and Teucrium yemense from Yemen.

\begin{tabular}{|c|c|c|c|c|c|}
\hline \multirow{2}{*}{$\mathrm{RI}_{\text {calc }}{ }^{\mathrm{a}}$} & \multirow{2}{*}{$\mathrm{RI}_{\text {lit }} \mathrm{b}$} & \multirow{2}{*}{ Compound } & \multicolumn{3}{|c|}{$\%$ Composition } \\
\hline & & & EOOF & EOTY-d & EOTY-t \\
\hline 936 & 930 & $\alpha$-Thujene & - & $\operatorname{tr}^{\mathrm{c}}$ & $\operatorname{tr}$ \\
\hline 942 & 939 & $\alpha$-Pinene & 0.5 & 2.3 & 6.6 \\
\hline 954 & 954 & Camphene & 0.3 & - & 0.1 \\
\hline 959 & 960 & Thuja-2,4(10)-diene & - & - & $\operatorname{tr}$ \\
\hline 976 & 975 & Sabinene & $\operatorname{tr}$ & 0.5 & $\operatorname{tr}$ \\
\hline 979 & 979 & $\beta$-Pinene & $\operatorname{tr}$ & 1.1 & 3.1 \\
\hline 982 & 979 & 1-Octen-3-ol & $\operatorname{tr}$ & - & - \\
\hline 992 & 990 & Myrcene & 0.4 & - & 0.2 \\
\hline 1004 & 1002 & $\alpha$-Phellandrene & $\operatorname{tr}$ & - & - \\
\hline 1009 & 1005 & (3Z)-Hexenyl acetate & $\operatorname{tr}$ & - & - \\
\hline 1025 & 1024 & $p$-Cymene & 0.1 & $\operatorname{tr}$ & $\operatorname{tr}$ \\
\hline 1028 & 1029 & Limonene & 2.5 & 0.5 & 1.2 \\
\hline 1031 & 1031 & 1,8-Cineole & 0.3 & - & - \\
\hline 1067 & 1070 & cis-Sabinene hydrate & 0.3 & - & - \\
\hline 1073 & 1068 & 1-Octanol & $\operatorname{tr}$ & - & - \\
\hline 1088 & 1086 & Fenchone & 12.2 & 0.3 & - \\
\hline 1094 & 1092 & 6,7-Epoxymyrcene & 0.1 & - & - \\
\hline 1100 & 1096 & Linalool & 5.7 & 0.2 & 0.1 \\
\hline 1113 & 1116 & endo-Fenchol & 31.1 & 0.1 & - \\
\hline 1122 & 1122 & trans-Pinene hydrate & 0.1 & - & - \\
\hline 1125 & 1127 & Chrysanthenone & - & $\operatorname{tr}$ & - \\
\hline 1126 & 1126 & $\alpha$-Campholenal & - & $\operatorname{tr}$ & 0.1 \\
\hline 1137 & 1140 & Nopinone & - & $\operatorname{tr}$ & - \\
\hline 1138 & 1139 & trans-Pinocarveol & - & 0.4 & 0.2 \\
\hline
\end{tabular}


Table 1. Cont.

\begin{tabular}{|c|c|c|c|c|c|}
\hline \multirow{2}{*}{$\mathrm{RI}_{\text {calc }}{ }^{\mathrm{a}}$} & \multirow{2}{*}{$\mathrm{RI}_{\text {lit }} \mathrm{b}$} & \multirow{2}{*}{ Compound } & \multicolumn{3}{|c|}{$\%$ Composition } \\
\hline & & & EOOF & EOTY-d & EOTY-t \\
\hline 1141 & 1141 & cis-Verbenol & - & $\operatorname{tr}$ & 0.1 \\
\hline 1144 & 1144 & trans-Verbenol & - & 1.0 & 0.3 \\
\hline 1145 & 1146 & Camphor & 6.2 & - & - \\
\hline 1157 & 1159 & Sabina ketone & - & $\operatorname{tr}$ & - \\
\hline 1162 & 1164 & Pinocarvone & - & - & $\operatorname{tr}$ \\
\hline 1165 & 1169 & Borneol & 1.0 & - & $\operatorname{tr}$ \\
\hline 1167 & 1169 & $p$-Mentha-1,5-dien-8-ol & - & - & 0.1 \\
\hline 1177 & 1177 & Terpinen-4-ol & 0.2 & 0.2 & $\operatorname{tr}$ \\
\hline 1183 & 1182 & $p$-Methylacetophenone & $\operatorname{tr}$ & $\operatorname{tr}$ & - \\
\hline 1185 & 1182 & p-Cymen-8-ol & 0.2 & 0.1 & 0.1 \\
\hline 1190 & 1188 & $\alpha$-Terpineol & 0.8 & 0.1 & 0.1 \\
\hline 1195 & 1195 & Myrtenal & - & 0.4 & 0.2 \\
\hline 1196 & 1195 & Myrtenol & - & - & $\operatorname{tr}$ \\
\hline 1198 & 1196 & Estragole (=Methyl chavicol) & 0.2 & - & - \\
\hline 1207 & 1205 & Verbenone & - & 0.6 & 0.2 \\
\hline 1217 & 1216 & trans-Carveol & - & $\operatorname{tr}$ & 0.1 \\
\hline 1219 & 1220 & endo-Fenchyl acetate & 2.8 & - & - \\
\hline 1237 & 1241 & Cuminaldehyde & - & $\operatorname{tr}$ & - \\
\hline 1241 & 1243 & Carvone & - & $\operatorname{tr}$ & $\operatorname{tr}$ \\
\hline 1243 & 1244 & Carvacrol methyl ether & - & - & $\operatorname{tr}$ \\
\hline 1252 & 1252 & Geraniol & $\operatorname{tr}$ & - & - \\
\hline 1285 & 1288 & Bornyl acetate & 0.1 & 0.4 & 0.3 \\
\hline 1299 & 1298 & trans-Pinocarvyl acetate & - & $\operatorname{tr}$ & - \\
\hline 1302 & 1299 & Carvacrol & $\operatorname{tr}$ & - & 0.2 \\
\hline 1305 & 1299 & Methyl (Z)-cinnamate & 0.9 & - & - \\
\hline 1324 & 1326 & Myrtenyl acetate & - & 1.0 & - \\
\hline 1334 & 1338 & $\delta$-Elemene & - & - & 0.1 \\
\hline 1349 & 1348 & $\alpha$-Cubebene & 0.1 & $\operatorname{tr}$ & 0.1 \\
\hline 1375 & 1376 & $\alpha$-Copaene & 0.1 & $\operatorname{tr}$ & 0.6 \\
\hline 1385 & 1378 & Methyl $(E)$-cinnamate & 5.1 & $\operatorname{tr}$ & - \\
\hline 1387 & 1388 & $\beta$-Bourbonene & - & 0.3 & 1.0 \\
\hline 1390 & 1388 & $\beta$-Cubebene & 0.2 & - & 0.2 \\
\hline 1393 & 1390 & $\beta$-Elemene & 0.3 & 0.9 & 0.3 \\
\hline 1406 & 1403 & Methyl eugenol & $\operatorname{tr}$ & - & - \\
\hline 1408 & 1408 & (Z)-Caryophyllene & - & $\operatorname{tr}$ & 0.1 \\
\hline 1409 & 1409 & $\alpha$-Gurjunene & - & - & 0.2 \\
\hline 1416 & 1412 & $\alpha$-cis-Bergamotene & $\operatorname{tr}$ & - & - \\
\hline 1419 & 1419 & (E)-Caryophyllene & 1.1 & 11.2 & 19.1 \\
\hline 1429 & 1432 & $\beta$-Copaene & - & - & 0.2 \\
\hline
\end{tabular}


Table 1. Cont.

\begin{tabular}{|c|c|c|c|c|c|}
\hline \multirow{2}{*}{$\mathrm{RI}_{\text {calc }}{ }^{\mathrm{a}}$} & \multirow{2}{*}{$\mathrm{RI}_{\text {lit }} \mathrm{b}$} & \multirow{2}{*}{ Compound } & \multicolumn{3}{|c|}{$\%$ Composition } \\
\hline & & & EOOF & EOTY-d & EOTY-t \\
\hline 1437 & 1434 & $\alpha$-trans-Bergamotene & 3.1 & 0.1 & $\operatorname{tr}$ \\
\hline 1439 & 1439 & $\alpha$-Guaiene & 0.4 & $\operatorname{tr}$ & $\operatorname{tr}$ \\
\hline 1444 & 1441 & Aromadendrene & - & - & $\operatorname{tr}$ \\
\hline 1454 & 1454 & $\alpha$-Humulene & 0.2 & 4.0 & 6.4 \\
\hline 1459 & 1456 & $(E)$ - $\beta$-Farnesene & 0.1 & 0.1 & 0.1 \\
\hline 1461 & 1454 & Alloaromadendrene & - & $\operatorname{tr}$ & 2.2 \\
\hline 1464 & 1466 & cis-Muurola-4(14),5-diene & 0.5 & - & 0.1 \\
\hline 1467 & 1467 & Ethyl $(E)$-cinnamate & - & $\operatorname{tr}$ & - \\
\hline 1474 & 1479 & trans-Cadina-1(6),4-diene & - & - & 0.2 \\
\hline 1476 & 1477 & $\gamma$-Gurjunene & - & $\operatorname{tr}$ & - \\
\hline 1478 & 1479 & $\gamma$-Muurolene & - & - & 0.4 \\
\hline 1482 & 1485 & Germacrene-D & 0.8 & 0.1 & 0.4 \\
\hline 1484 & $1479^{\mathrm{d}}$ & $\gamma$-Selinene & - & 5.5 & 0.4 \\
\hline 1487 & 1490 & $\beta$-Selinene & 1.2 & 0.3 & 2.5 \\
\hline 1495 & 1494 & epi-Cubebol & - & - & 0.9 \\
\hline 1495 & 1496 & Valencene & - & 3.7 & - \\
\hline 1496 & 1492 & $\delta$-Selinene & 0.8 & - & - \\
\hline 1497 & 1500 & Bicyclogermacrene & - & - & 0.8 \\
\hline 1500 & 1496 & Viridiflorene & 0.1 & - & - \\
\hline 1502 & 1500 & $\alpha$-Muurolene & - & $\operatorname{tr}$ & 1.0 \\
\hline 1506 & 1509 & Germacrene A & - & - & 0.1 \\
\hline 1507 & 1509 & $\alpha$-Bulnesene & 0.9 & - & - \\
\hline 1510 & 1505 & $\beta$-Bisabolene & - & $\operatorname{tr}$ & 0.1 \\
\hline 1516 & 1513 & $\gamma$-Cadinene & 2.9 & - & 2.7 \\
\hline 1521 & 1522 & 7-epi- $\alpha$-Selinene & 0.1 & 20.1 & 1.3 \\
\hline 1523 & 1522 & trans-Calamenene & 0.2 & - & - \\
\hline 1525 & 1523 & $\delta$-Cadinene & 0.2 & 0.4 & 6.5 \\
\hline 1532 & 1535 & 10-epi-Cubebol & 0.1 & - & - \\
\hline 1534 & 1534 & trans-Cadina-1,4-diene & - & - & 0.1 \\
\hline 1537 & 1538 & $\alpha$-Cadinene & $\operatorname{tr}$ & - & 0.2 \\
\hline 1547 & 1544 & cis-Sesquisabinene hydrate & - & 3.4 & 0.9 \\
\hline 1553 & - & Unidentified & - & 1.2 & - \\
\hline 1557 & 1561 & cis-Muurol-5-en- $4 \alpha$-ol & 0.1 & - & - \\
\hline 1560 & 1563 & (E)-Nerolidol & - & $\operatorname{tr}$ & - \\
\hline 1565 & 1565 & $\beta$-Calacorene & - & $\operatorname{tr}$ & - \\
\hline 1566 & - & Unidentified & - & 0.5 & - \\
\hline 1578 & 1575 & Germacrene D-4-ol & - & - & 3.1 \\
\hline 1584 & 1583 & Caryophyllene oxide & 0.2 & 20.1 & 4.3 \\
\hline 1604 & 1602 & Ledol & - & 3.6 & 0.5 \\
\hline
\end{tabular}


Table 1. Cont.

\begin{tabular}{|c|c|c|c|c|c|}
\hline \multirow{2}{*}{$\mathrm{RI}_{\text {calc }}{ }^{\mathrm{a}}$} & \multirow{2}{*}{$\mathrm{RI}_{\text {lit }} \mathrm{b}$} & \multirow{2}{*}{ Compound } & \multicolumn{3}{|c|}{$\%$ Composition } \\
\hline & & & EOOF & EOTY-d & EOTY-t \\
\hline 1610 & 1608 & Humulene epoxide II & - & - & 0.9 \\
\hline 1616 & 1619 & 1,10-di-epi-Cubenol & 1.6 & - & 0.1 \\
\hline 1619 & 1623 & 10-epi- $\gamma$-Eudesmol & - & 0.6 & 0.3 \\
\hline 1629 & 1628 & 1-epi-Cubenol & - & 0.6 & 0.6 \\
\hline 1637 & 1640 & Caryophylla-4(12),8(13)-dien-5-ol & - & 0.7 & 0.4 \\
\hline 1643 & 1640 & $\tau$-Cadinol & 12.2 & 2.0 & 5.7 \\
\hline 1646 & 1642 & $\tau$-Muurolol & - & 0.6 & 4.9 \\
\hline 1647 & 1646 & $\alpha$-Muurolol (= Torreyol) & - & - & 0.7 \\
\hline 1648 & 1649 & Methyl (Z)-jasmonate & 0.2 & - & - \\
\hline 1653 & 1650 & $\beta$-Eudesmol & 0.1 & 0.9 & 0.3 \\
\hline 1657 & 1654 & $\alpha$-Cadinol & 0.4 & 2.0 & 9.5 \\
\hline 1660 & $\begin{array}{l}1663 \\
1666 \\
1661 \\
\end{array}$ & $\begin{array}{c}\text { 7-epi- } \alpha \text {-Eudesmol } \\
\quad+\text { Intermediol } \\
+ \text { cis-Calamenen-10-ol }\end{array}$ & - & 3.3 & - \\
\hline 1668 & 1669 & trans-Calamenen-10-ol & - & 0.6 & - \\
\hline 1672 & 1671 & epi- $\beta$-Bisabolol & - & - & 0.3 \\
\hline 1672 & 1669 & 14-Hydroxy-9-epi-(E)-caryophyllene & e - & 0.8 & - \\
\hline 1674 & 1676 & Cadalene & - & 0.4 & - \\
\hline 1685 & 1685 & $\alpha$-Bisabolol & 0.1 & - & - \\
\hline 1687 & 1689 & cis-14-nor-Muurol-5-en-4-one & 0.3 & - & - \\
\hline 1690 & 1689 & Shyobunol & - & - & 4.6 \\
\hline 1704 & 1702 & 10-Norcalamenen-10-one & - & 0.2 & - \\
\hline 1738 & 1740 & Oplopanone & - & 0.3 & $\operatorname{tr}$ \\
\hline 1773 & 1775 & epi-Cyclocolorenone & - & 0.8 & - \\
\hline \multirow[t]{2}{*}{1803} & 1806 & Nootkatone & - & 0.6 & - \\
\hline & & Total Identified & 100 & 91.7 & 98.6 \\
\hline
\end{tabular}

a $\mathrm{RI}_{\text {calc }}=$ Retention Index calculated with respect to a homologous series of $n$-alkanes on an HP-5ms column; ${ }^{\mathrm{b}} \mathrm{RI}_{\mathrm{lit}}=$ Retention Index from Adams [14]; ${ }^{\mathrm{c}} \mathrm{tr}=$ trace $(<0.05 \%)$; EOOF = Ocimum forskolei Benth.; EOTY- $\mathrm{d}=$ Teucrium yemense Deflers collected from Dhamar province; EOTY- $\mathrm{t}=$ Teucrium yemense Deflers collected from Taiz; ${ }^{\mathrm{d}}$ National Institute of Standards and Technology (NIST).

\subsubsection{Teucrium yemense}

The essential oil compositions of two different collections of $T$. yemense are compiled in Table 1. Although there have been several studies on Teucrium essential oils, T. yemense has not been extensively studied, possibly due to its confined availability. In a previous study, T. yemense collected from Taiz (Sabir Mountain, altitude $1300 \mathrm{~m}$ ) showed a total of 12 identifiable compounds, the major being $\delta$-cadinene $(34.9 \%),(E)$-caryophyllene $(22.7 \%), \alpha$-humulene $(6.1 \%)$, and $\alpha$-selinene $(5.4 \%)$, as well as two unidentified sesquiterpenes (16.5\%) [12]. In this current work, two different populations of T. yemense were examined, one collected in May 2010 from Dhamar province, and another collected in February, 2012 from Taiz. Although qualitatively similar, there were notable quantitative differences between these two collections and a previous collection from Taiz in 2005 [12]. All three collections showed $(E)$-caryophyllene to be a major component $(11.2 \%-22.7 \%)$. $\delta$-Cadinene was the major component from the Taiz sample in 2005 [12], but was only a minor component in the Dhamar 
sample, while 7-epi- $\alpha$-selinene (20.1\%) and caryophyllene oxide (20.1\%) were the major components in the Dhamar sample. $\alpha$-Humulene was relatively abundant in all three T. yemense samples $(4.0 \%-6.4 \%)$, while $\alpha$-cadinol was relatively abundant (2.0 and 9.5\%) in the two T. yemense samples in this current investigation. Sesquiterpenoids, including $(E)$-caryophyllene, $\alpha$-humulene, germacrene $D, \delta$-cadinene, caryophyllene oxide, $\alpha$-cadinol, and $\tau$-cadinol, have been generally abundant in Teucrium essential oils (Table 2) [24-45]. Although relatively abundant in T. yemense, 7-epi- $\alpha$-selinene has been found in relatively small amounts in T. persicum [46] and T. capitatum [33].

Table 2. Major sesquiterpenoid components found in Teucrium essential oils.

\begin{tabular}{|c|c|c|c|}
\hline Major Sesquiterpenoid & Teucrium Species & $\%$ & Refs. \\
\hline \multirow{5}{*}{ (E)-Caryophyllene } & T. chamaedrys & 47.6 & {$[24]$} \\
\hline & T. polium & 52.0 & {$[24]$} \\
\hline & T. arduini & 35.2 & [25] \\
\hline & T. turredanum & $15.6-32.6$ & [26] \\
\hline & T. scorodonia ssp. baeticum & 33.8 & [27] \\
\hline \multirow{6}{*}{$\alpha$-Humulene } & T. alopecurus & 12.3 & [28] \\
\hline & T. flavum & 8.4 & [29] \\
\hline & T. marum & 7.2 & [30] \\
\hline & T. polium & 4.3 & [31] \\
\hline & T. scorodonia ssp. baeticum & 9.1 & [27] \\
\hline & T. turredanum & $4.7-10.1$ & [26] \\
\hline \multirow{4}{*}{ Germacrene $\mathrm{D}^{\mathrm{a}}$} & T. sandrasicum & 27.9 & [42] \\
\hline & T. arduini & $17.0-18.7$ & {$[25,43]$} \\
\hline & T. chamaedrys & $16.5-32.1$ & {$[24,44,45]$} \\
\hline & T. scorodonia ssp. baeticum & 22.2 & [27] \\
\hline \multirow{5}{*}{$\delta$-Cadinene } & T. montanum & 17.2 & [32] \\
\hline & T. capitatum & $3.0-9.8$ & [33] \\
\hline & T. maghrebinum & 13.5 & {$[34]$} \\
\hline & T. ramosissimum & 20.0 & [35] \\
\hline & T. stocksianum & 12.9 & [36] \\
\hline \multirow{3}{*}{ Caryophyllene oxide } & T. orientale ssp. taylori & 15.6 & [37] \\
\hline & T. montbretti & 12.7 & [38] \\
\hline & T. arduini & 14.6 & [39] \\
\hline \multirow{5}{*}{$\alpha$-Cadinol } & T. polium ssp. aurasiacum & 46.8 & [40] \\
\hline & T. polium ssp. capitatum & 4.5 & [38] \\
\hline & T. ramosissimum & 9.9 & {$[35]$} \\
\hline & T. leucocladum & 9.3 & {$[41]$} \\
\hline & T. capitatum & $1.6-9.8$ & [33] \\
\hline \multirow{3}{*}{$\tau$-Cadinol } & T. capitatum & $1.6-24.1$ & [33] \\
\hline & T. montanum & 5.5 & [42] \\
\hline & T. leucocladum & 5.5 & [41] \\
\hline
\end{tabular}

${ }^{\text {a }}$ Not abundant in T. yemense essential oils.

\subsection{Biological Activities}

\subsubsection{Free Radical Scavenging}

O. forskolei and T. yemense leaf oils from Dhamar (2010) were screened for free-radical-inhibitory activity using the DPPH. radical scavenging assay. Neither essential oil showed remarkable radical inhibition $\left(\mathrm{IC}_{50}=31.55\right.$ and $31.41 \mu \mathrm{L} / \mathrm{mL}$, respectively). This is not surprising; neither essential oil has high concentrations of phenolic compounds such a carvacrol, thymol, or eugenol, which are known to be excellent radical scavenging agents [47]. endo-Fenchol, the major component in O. forskolei oil, (E)-caryophyllene and $\alpha$-humulene, major components in T. yemense oil, have shown only weak antioxidant activities [48]. 


\subsubsection{Antimicrobial Activity}

O. forskolei and T. yemense essential oils were screened for antibacterial and antifungal activity (Table 3). The disc diffusion assay showed O. forskolei to have weak antibacterial activity against Staphylococcus aureus, but stronger activity against Bacillus subtilis with an inhibition zone of $34 \mathrm{~mm}$, and antifungal activity against Candida albicans with an inhibition zone of $24 \mathrm{~mm}$. EOTY-d was virtually devoid of antimicrobial activity. Using the broth microdilution assay, EOTY-t showed antibacterial activity against $S$. aureus and B. cereus (MIC $=156 \mu \mathrm{g} / \mathrm{mL}$ ), as well as antifungal activity against Aspergillus niger and Botrytis cinerea with MIC of $313 \mu \mathrm{g} / \mathrm{mL}$.

Table 3. Antimicrobial activity (minimum inhibitory concentration (MIC), $\mu \mathrm{g} / \mathrm{mL}$ ) of Ocimum forskolei and Teucrium yemense leaf essential oils.

\begin{tabular}{cccc}
\hline Organism & EOOF $^{\mathbf{a}}$ & EOTY-t $^{\mathbf{b}}$ & Positive Control $^{\mathrm{a}}$ \\
\hline S. aureus & 4300 & 156 & $0.61^{\mathrm{e}}$ \\
MRSA & 8600 & $\mathrm{nt}^{\mathrm{c}}$ & $<10.0^{\mathrm{f}}$ \\
B. cereus & $\mathrm{nt}$ & 156 & $1.22^{\mathrm{e}}$ \\
B. subtilis & 4300 & $\mathrm{nt}$ & $<10.0^{\mathrm{e}}$ \\
E. coli & $\mathrm{na} \mathrm{d}$ & 313 & $1.22^{\mathrm{e}}$ \\
P. aeruginosa & $\mathrm{na}$ & 1250 & $2.44^{\mathrm{e}}$ \\
C. albicans & 8600 & 1250 & $0.61^{\mathrm{g}}$ \\
A. niger & $\mathrm{nt}$ & 313 & $0.61^{\mathrm{g}}$ \\
B. cinerea & $\mathrm{nt}$ & 313 & $<19.5^{\mathrm{g}}$
\end{tabular}

a Antimicrobial activity of $O$. forskolei essential oil determined using the disc diffusion assay; ${ }^{\mathrm{b}}$ Antimicrobial activity determined using the broth dilution assay on T. yemense Taiz (2012) essential oil; ${ }^{c}$ "nt" = not tested; ${ }^{\mathrm{d}}$ "na" = not active (no zone of inhibition); ${ }^{e}$ Bacterial positive control, gentamicin sulfate; ${ }^{\mathrm{f}}$ MRSA positive control, enoxacin; $\mathrm{g}$ Fungal positive control, amphotericin B.

The antibacterial activity of $O$. forskolei essential oil against B. subtilis and S. aureus is likely due to methyl $(E)$-cinnamate and $\tau$-cadinol. Methyl cinnamate has shown antibacterial activity against S. aureus [49] while $\tau$-cadinol has shown activity against B. cereus and S. aureus [50]. Apparently neither fenchone nor fenchol are antibacterial [51] and fenchone is also not antifungal [52].

The antibacterial activity of EOTY-t essential oil against S. aureus, B. cereus, E. coli, and A. niger can be attributed to the relatively high concentrations of $(E)$-caryophyllene, $\alpha$-humulene, $\delta$-cadinene, and $\alpha$-cadinol in the oil. (E)-Caryophyllene has shown antibacterial activity against each of these organisms [53,54]. $\alpha$-Humulene has shown antibacterial activity against $B$. cereus and S. aureus [53] as well as antifungal activity against $A$. niger [53]. $\delta$-Cadinene was shown to be antibacterial against Bacillus subtilis and Propionibacterium acnes [55,56], while $\alpha$-cadinol has shown activity against $B$. cereus and S. aureus [50].

\subsubsection{Cytotoxic Activity}

O. forskolei leaf oil was screened for cytotoxic activity against HT-29 human colorectal adenocarcinoma cells, but was inactive (Table 4). EOTY-d was active against this cell line with $\mathrm{IC}_{50}=43.7 \mu \mathrm{g} / \mathrm{mL}$. Consistent with this, EOTY-t was active against both MCF-7 and MDA-MB-231 human breast adenocarcinoma cells.

Table 4. Cytotoxic activity $\left(\mathrm{IC}_{50}, \mu \mathrm{g} / \mathrm{mL}\right)$ of Ocimum forskolei and Teucrium yemense leaf essential oils.

\begin{tabular}{cccc}
\hline Cell Line & EOOF & EOTY-d & EOTY-t \\
\hline HT-29 & $\mathrm{na}^{\mathrm{a}}$ & $43.7 \pm 7.1$ & $\mathrm{nt}^{\mathrm{b}}$ \\
MCF-7 & $\mathrm{nt}$ & $\mathrm{nt}$ & $24.4 \pm 1.8$ \\
MDA-MB-231 & $\mathrm{nt}$ & $\mathrm{nt}$ & $59.9 \pm 4.6$ \\
\hline a "na" = not active $(0 \%$ kill at $100 \mu \mathrm{g} / \mathrm{mL}){ }^{\text {b }}$ "nt" = not tested.
\end{tabular}


The cytotoxic activities of $T$. yemense leaf essential oils is likely due to the relatively high concentrations of known cytotoxic components such as (E)-caryophyllene (MCF-7 [57], HT-29 [58,59], other cell lines [56]) $\alpha$-humulene (MCF-7 [57], HT-29 [58,59], other cell lines [60,61]) $\delta$-cadinene [56], caryophyllene oxide (MCF-7 [57], other cell lines [62]), and $\alpha$-cadinol (MCF-7, HT-29 [63], other cell lines [50]). Furthermore, $(E)$-caryophyllene has been shown to potentiate the cytotoxic activities of other sesquiterpenoids [64]. Interestingly, although O. forskolei essential oil was not cytotoxic, one of its major components, $\tau$-cadinol, has shown cytotoxic activity [50]. Fenchone, however, has been shown not to be cytotoxic [57], and there are apparently no reports in the literature about cytotoxicity of endo-fenchol.

\section{Conclusions}

In summary, this preliminary phytochemical and bioactivity investigation has described the composition of Ocimum forskolei leaf essential oil from Yemen for the first time, and has shown the leaf oil to present moderate antimicrobial properties against B. subtilis and C. albicans. This activity is consistent with its uses in Yemeni traditional medicine for some skin infections. The variation in the chemical compositions and biological activities of $T$. yemense leaf essential oils can be due to the different environmental factors such as altitude, latitude, or time of collection. Both populations of T. yemense showed good cytotoxic activity. There remains the need to follow up this preliminary study and investigate both species more comprehensively in terms of botanical, genetic, phytochemical, and biological properties. This is particularly important because much primary health care in Yemen still relies on traditional herbal practices.

Acknowledgments: N.A.A.A. is grateful to the Deutscher Akademischer Austauschdienst (DAAD) for a grant enabling his stay at the Leibniz Institute of Plant Biochemistry Halle/S, Germany. W.N.S. thanks an anonymous private donor for the generous gift of the GC-MS instrumentation.

Author Contributions: N.A.A.A. conceived and designed the experiments; B.K.C., N.S.D., K.S., and A.J.A.A.-F performed the experiments; B.K.C., N.A.A.A., and W.N.S. analyzed the data; L.W. and W.N.S. contributed reagents/materials/analysis tools; N.A.A.A. and W.N.S. wrote the paper.

Conflicts of Interest: The authors declare no conflict of interest.

\section{References}

1. Wood, J.R.I. A Handbook of the Yemen Flora; Royal Botanic Gardens: Kew, UK, 1997.

2. Mabberley, D.J. Mabberley's Plant-Book, 3rd ed.; Cambridge University Press: Cambridge, UK, 2008.

3. Ryding, O. Notes on the Sweet Basil and Its Wild Relatives (Lamiaceae). Econ. Bot. 1994, 48, 65-67. [CrossRef]

4. Hall, M.; Al-Khulaidi, A.W.; Miller, A.G.; Scholte, P.; Al-Qadasi, A.H. Arabia's last forests under threat: Plant biodiversity and conservation in the valley forest of Jabal Bura (Yemen). Edinb. J. Bot. 2008, 65, 113-135. [CrossRef]

5. Hammer, K.; Gebauer, J.; Al Khanjari, S.; Buerkert, A. Oman at the cross-roads of inter-regional exchange of cultivated plants. Genet. Resour. Crop Evol. 2009, 56, 547-560. [CrossRef]

6. Sakkir, S.; Kabshawi, M.; Mehairbi, M. Medicinal plants diversity and their conservation status in the United Arab Emirates (UAE). J. Med. Plants Res. 2012, 6, 1304-1322.

7. Al-Fatimi, M.; Wurster, M.; Schröder, G.; Lindequist, U. Antioxidant, antimicrobial and cytotoxic activities of selected medicinal plants from Yemen. J. Ethnopharmacol. 2007, 111, 657-666. [CrossRef] [PubMed]

8. Dekker, T.; Ignell, R.; Ghebru, M.; Glinwood, R.; Hopkins, R. Identification of mosquito repellent odours from Ocimum forskolei. Parasites Vectors 2011, 4. [CrossRef] [PubMed]

9. Al-Hajj, N.Q.M.; Wang, H.X.; Ma, C.; Lou, Z.; Bashari, M.; Thabit, R. Antimicrobial and antioxidant activities of the essential oils of some aromatic medicinal plants (Pulicaria inuloides-Asteraceae and Ocimum forskolei-Lamiaceae). Trop. J. Pharmaceut. Res. 2014, 13, 1287-1293. [CrossRef]

10. Onifade, A.K. Effect of essential oils from five Ocimum sp. on the pathogenicity of Pretylenchus brachyurus (Godfrey) in tomato. Agric. J. 2007, 2, 185-191. 
11. Fatope, M.O.; Marwah, R.G.; Al Hadhrami, N.M.; Onifade, A.K.; Williams, J.R. Identification of the chemotypes of Ocimum forskolei and Ocimum basilicum by NMR spectroscopy. Chem. Biodivers. 2008, 5, 2457-2463. [CrossRef] [PubMed]

12. Ali, N.A.A.; Wurster, M.; Arnold, N.; Lindequist, U.; Wessjohann, L. Chemical composition of the essential oil of Teucrium yemense Deflers. Rec. Nat. Prod. 2008, 2, 25-32.

13. Coucil of Europe. European Pharmacopoeia, 3rd ed.; Council of Europe Press: Strasbourg, France, 1997.

14. Adams, R.P. Identification of Essential Oil Components by Gas Chromatography/Mass Spectrometry, 4th ed.; Allured Publishing: Carol Stream, IL, USA, 2007.

15. Mohamad, H.; Abas, F.; Permana, D.; Lajis, N.H.; Ali, A.M.; Sukari, M.A.; Hin, T.Y.Y.; Kikuzaki, H.; Nakatani, N. DPPH free radical ccavenger components from the fruits of Alpinia rafflesiana Wall. Ex. Bak. (Zingiberaceae). Z. Naturforsch. C 2004, 59, 811-815. [CrossRef] [PubMed]

16. Ali, N.A.A.; Sharopov, F.S.; Alhaj, M.; Hill, G.M.; Porzel, A.; Arnold, N.; Setzer, W.N.; Schmidt, J.; Wessjohann, L. Chemical composition and biological activity of essential oil from Pulicaria undulata from Yemen. Nat. Prod. Commun. 2012, 7, 257-260. [PubMed]

17. Setzer, M.C.; Setzer, W.N.; Jackes, B.R.; Gentry, G.A.; Moriarity, D.M. The medicinal value of tropical rainforest plants from Paluma, North Queensland, Australia. Pharmaceut. Biol. 2001, 39, 67-78. [CrossRef]

18. Scudiero, D.A.; Shoemaker, R.H.; Paull, K.D.; Monks, A.; Tierney, S.; Nofziger, T.H.; Currens, M.J.; Seniff, D.; Boyd, M.R. Evaluation of a soluble tetrazolium/formazan assay for cell growth and drug sensitivity in culture using human and other tumor cell lines. Cancer Res. 1988, 48, 4827-4833. [PubMed]

19. Johansson, S.; Lindholm, P.; Gullbo, J.; Larsson, R.; Bohlin, L.; Claeson, P. Cytotoxicity of digitoxin and related cardiac glycosides in human tumor cells. Anti-Cancer Drugs 2001, 12, 475-483. [CrossRef] [PubMed]

20. Berridge, M.V.; Herst, P.M.; Tan, A.S. Tetrazolium dyes as tools in cell biology: New insights into their cellular reduction. Biotechnol. Annu. Rev. 2005, 11, 127-152.

21. Setzer, W.N.; Setzer, M.C.; Hopper, A.L.; Moriarity, D.M.; Lehrman, G.K.; Niekamp, K.L.; Morcomb, S.M.; Bates, R.B.; McClure, K.J.; Stessman, C.C.; et al. The cytotoxic activity of a Salacia liana species from Monteverde, Costa Rica, is due to a high concentration of tingenone. Planta Med. 1998, 64, 583. [CrossRef] [PubMed]

22. Ferrari, M.; Fornasiero, C.M.; Isetta, A.M. MTT Colorimetric assay for testing macrophage cytotoxic activity in vitro. J. Immunol. Meth. 1990, 131, 165-172. [CrossRef]

23. Wood, J.R.I. The genus Ocimum (Labiatae) in Forsskal's Flora Aegyptiaco-Arabica. Kew Bull. 1983, 37, 597-603. [CrossRef]

24. Bezić, N.; Vuko, E.; Dunkić, V.; Ruščić, M.; Blažević, I.; Burčul, F. Antiphytoviral activity of sesquiterpene-rich essential oils from four Croatian Teucrium species. Molecules 2011, 16, 8119-8129. [CrossRef] [PubMed]

25. Kremer, D.; Košir, I.J.; Kosalec, I.; Končić, M.Z.; Potočnik, T.; Čerenak, A.; Bezić, N.; Srečec, S.; Dunkić, V. Investigation of chemical compounds, antioxidant and antimicrobial properties of Teucrium arduini L. (Lamiaceae). Curr. Drug Targets 2013, 14, 1006-1014. [CrossRef] [PubMed]

26. Blázquez, M.A.; Pérez, I.; Boira, H. Essential oil analysis of Teucrium libanitis and T. turredanum by GC and GC-MS. Flavour Fragr. J. 2003, 18, 497-501. [CrossRef]

27. Djabou, N.; Muselli, A.; Allali, H.; Dib, M.E.A.; Tabti, B.; Varesi, L.; Costa, J. Chemical and genetic diversity of two Mediterranean subspecies of Teucrium polium L. Phytochemistry 2012, 83, 51-62. [CrossRef] [PubMed]

28. Hachicha, S.F.; Skanji, T.; Barrek, S.; Zarrouk, H.; Ghrabi, Z.G. Chemical composition of Teucrium alopecurus essential oil from Tunisia. J. Essent. Oil Res. 2007, 19, 413-415. [CrossRef]

29. Baher, Z.F.; Mirza, M. Volatile constituents of Teucrium flavum L. from Iran. J. Essent. Oil Res. 2003, 15, 106-107. [CrossRef]

30. Ricci, D.; Fraternale, D.; Giamperi, L.; Bucchini, A.; Epifano, F.; Burini, G.; Curini, M. Chemical composition, antimicrobial and antioxidant activity of the essential oil of Teucrium marum (Lamiaceae). J. Ethnopharmacol. 2005, 98, 195-200. [CrossRef] [PubMed]

31. Aburjai, T.; Hudaib, M.; Cavrini, V. Composition of the essential oil from Jordanian germander (Teucrium polium L.). J. Essent. Oil Bear. Plants 2006, 18, 97-99. [CrossRef]

32. Vukovic, N.; Milosevic, T.; Sukdolak, S.; Solujic, S. Antimicrobial activities of essential oil and methanol extract of Teucrium montanum. Evid. Based Complement. Altern. Med. 2007, 4 (Suppl. 1), 17-20. [CrossRef] [PubMed] 
33. Antunes, T.; Sevinate-Pinto, I.; Barroso, J.G.; Cavaleiro, C.; Salgueiro, L.R. Micromorphology of trichomes and composition of essential oil of Teucrium capitatum. Flavour Fragr. J. 2004, 19, 336-340. [CrossRef]

34. De Martino, L.; Formisano, C.; Mancini, E.; de Feo, V.; Piozzi, F.; Rigano, D.; Senatore, F. Chemical composition and phytotoxic effects of essential oils from four Teucrium species. Nat. Prod. Commun. 2010, 5, 1969-1976. [PubMed]

35. Hachicha, S.F.; Skanji, T.; Barrek, S.; Ghrabi, Z.G.; Zarrouk, H. Composition of the essential oil of Teucrium ramosissimum Desf. (Lamiaceae) from Tunisia. Flavour Fragr. J. 2007, 22, 101-104. [CrossRef]

36. Shah, S.M.M.; Ullah, F.; Shah, S.M.H.; Zahoor, M.; Sadiq, A. Analysis of chemical constituents and antinociceptive potential of essential oil of Teucrium stocksianum Bioss collected from the north west of Pakistan. BMC Complement. Altern. Med. 2012, 12, 12-17. [CrossRef] [PubMed]

37. Amiri, H. Antioxidant activity of the essential oil and methanolic extract of Teucrium orientale (L.) subsp. taylori (Boiss.) Rech. F. Iran. J. Pharm. Res. 2010, 9, 417-423.

38. Menichini, F.; Conforti, F.; Rigano, D.; Formisano, C.; Piozzi, F.; Senatore, F. Phytochemical composition, anti-inflammatory and antitumour activities of four Teucrium essential oils from Greece. Food Chem. 2009, 115, 679-686. [CrossRef]

39. Dunkić, V.; Bezić, N.; Vuko, E. Antiphytoviral activity of essential oil from endemic species Teucrium arduini. Nat. Prod. Commun. 2011, 6, 1385-1388. [PubMed]

40. Kabouche, A.; Kabouche, Z.; Ghannadi, A.; Sajjadi, S.E. Analysis of the essential oil of Teucrium polium ssp. aurasiacum from Algeria. J. Essent. Oil Res. 2007, 19, 44-46. [CrossRef]

41. El-Shazly, A.M.; Hussein, K.T. Chemical analysis and biological activities of the essential oil of Teucrium leucocladum Boiss. (Lamiaceae). Biochem. Syst. Ecol. 2004, 32, 665-674. [CrossRef]

42. Baser, K.H.C.; Demirçakmak, B. Composition of the essential oils of three Teucrium species from Turkey. J. Essent. Oil Res. 1997, 9, 545-549. [CrossRef]

43. Vukovic, N.; Sukdolak, S.; Solujic, S.; Mihailovic, V.; Mladenovic, M.; Stojanovic, J.; Stankovic, M.S. Chemical composition and antimicrobial activity of Teucrium arduini essential oil and cirsimarin from Montenegro. J. Med. Plants Res. 2011, 5, 1244-1250.

44. Morteza-Semnani, K.; Akbarzadeh, M.; Rostami, B. The essential oil composition of Teucrium chamaedrys L. from Iran. Flavour Fragr. J. 2005, 20, 544-546. [CrossRef]

45. Bagci, E.; Yazgın, A.; Hayta, S.; Cakılcıglu, U. Composition of the essential oil of Teucrium chamaedrys L. (Lamiaceae) from Turkey. J. Med. Plants Res. 2010, 4, 2587-2589.

46. Javidnia, K.; Miri, R.; Khosravi, A.R. Composition of the essential oil of Teucrium persicum from Iran. J. Essent. Oil Res. 2007, 19, 430-432. [CrossRef]

47. Sharopov, F.S.; Wink, M.; Setzer, W.N. Radical scavenging and antioxidant activities of essential oil components-An experimental and computational investigation. Nat. Prod. Commun. 2015, 10, 153-156. [PubMed]

48. Ruberto, G.; Baratta, M.T. Antioxidant activity of selected essential oil components in two lipid model systems. Food Chem. 2000, 69, 167-174. [CrossRef]

49. Gilles, M.; Zhao, J.; An, M.; Agboola, S. Chemical composition and antimicrobial properties of essential oils of three Australian Eucalyptus species. Food Chem. 2010, 119, 731-737. [CrossRef]

50. Su, Y.-C.; Ho, C.-L. Composition of the leaf essential oil of Phoebe formosana from Taiwan and its in vitro cytotoxic, antibacterial, and antifungal activities. Nat. Prod. Commun. 2016, 11, 845-848. [PubMed]

51. Kotan, R.; Kordali, S.; Cakir, A. Screening of antibacterial activities of twenty-one oxygenated monoterpenes. Z. Naturforsch. C 2007, 62, 507-513. [CrossRef] [PubMed]

52. Marei, G.I.K.; Abdel Rasoul, M.A.; Abdelgaleil, S.A.M. Comparative Antifungal Activities and Biochemical Effects of Monoterpenes on Plant Pathogenic Fungi. Pestic. Biochem. Physiol. 2012, 103, 56-61. [CrossRef]

53. Schmidt, J.M.; Noletto, J.A.; Vogler, B.; Setzer, W.N. Abaco bush medicine: Chemical composition of the essential oils of four aromatic medicinal plants from Abaco Island, Bahamas. J. Herbs Spices Med. Plants 2006, 12, 43-65. [CrossRef]

54. Dahham, S.S.; Tabana, Y.M.; Iqbal, M.A.; Ahamed, M.B.; Ezzat, M.O.; Majid, A.S.; Majid, A.M. The anticancer, antioxidant and antimicrobial properties of the sesquiterpene $\beta$-caryophyllene from the essential oil of Aquilaria crassna. Molecules 2015, 20, 11808-11829. [CrossRef] [PubMed]

55. Kubo, I.; Muroi, H.; Kubo, A. Naturally occurring antiacne agents. J. Nat. Prod. 1994, 57, 9-17. [CrossRef] [PubMed] 
56. Kubo, I.; Morimitsu, Y. Cytotoxicity of green tea flavor compounds against two solid tumor cells. J. Agric. Food Chem. 1995, 43, 1626-1628. [CrossRef]

57. Wright, B.S.; Bansal, A.; Moriarity, D.M.; Takaku, S.; Setzer, W.N. Cytotoxic leaf essential oils from Neotropical Lauraceae: Synergistic effects of essential oil components. Nat. Prod. Commun. 2007, 2, 1241-1244.

58. Da Silva, S.L.; Figueiredo, P.M.; Yano, T. Cytotoxic evaluation of essential oil from Zanthoxylum rhoifolium Lam. leaves. Acta Amazon. 2007, 37, 281-286. [CrossRef]

59. Su, Y.-C.; Hsu, K.-P.; Ho, C.-L. Composition, in vitro cytotoxicity, and anti-mildew activities of the leaf essential oil of Machilus thunbergii from Taiwan. Nat. Prod. Commun. 2015, 10, 2013-2016. [PubMed]

60. Sylvestre, M.; Pichette, A.; Lavoie, S.; Longtin, A.; Legault, J. Composition and cytotoxic activity of the leaf essential oil of Comptonia peregrina (L.) Coulter. Phytother. Res. 2007, 21, 536-540. [CrossRef] [PubMed]

61. Legault, J.; Dahl, W.; Debiton, E.; Pichette, A.; Madelmont, J.-C. Antitumor activity of balsam fir oil: Production of reactive oxygen species induced by $\alpha$-humulene as possible mechanism of action. Planta Med. 2003, 69, 402-407. [PubMed]

62. Jun, N.J.; Mosaddik, A.; Moon, J.Y.; Jang, K.C.; Lee, D.S.; Ahn, K.S.; Cho, S.K. Cytotoxic activity of $\beta$-caryophyllene oxide isolated from Jeju guava (Psidium cattleianum Sabine) leaf. Rec. Nat. Prod. 2011, 5, 242-246.

63. He, K.; Zeng, L.; Shi, G.; Zhao, G.X.; Kozlowski, J.F.; McLaughlin, J.L. Bioactive compounds from Taiwania cryptomerioides. J. Nat. Prod. 1997, 60, 38-40. [CrossRef] [PubMed]

64. Legault, J.; Pichette, A. Potentiating effect of $\beta$-caryophyllene on anticancer activity of $\alpha$-humulene, isocaryophyllene and paclitaxel. J. Pharm. Pharmacol. 2007, 59, 1643-1647. [CrossRef] [PubMed]

(C) 2017 by the authors. Licensee MDPI, Basel, Switzerland. This article is an open access article distributed under the terms and conditions of the Creative Commons Attribution (CC BY) license (http:/ / creativecommons.org/licenses/by/4.0/). 